\title{
Extracción de compuestos fenólicos y contenido de catequina en cortezas de tres especies forestales del Cauca-Colombia ${ }^{1}$
}

\section{Extraction of phenolic compounds and content of catechin in barks of three forest species from Cauca-Colombia}

\author{
R. A. Sarria-Villa, J. A. Gallo, R. Benítez \\ Recibido: marzo 19 de 2021 - Aceptado: junio 20 de 2021.
}

\begin{abstract}
Resumen-Se evaluó el contenido de catequina en cortezas de las especies forestales Pinus patula, Pinus oocarpa y Eucaliptus grandis, cultivadas en el Departamento del Cauca-Colombia. Los extractos etanólicos se obtuvieron evaluando las mejores condiciones de tamaño de partícula, tiempo de agitación, relación disolvente-corteza, temperatura y agitación. El contenido de catequina se determinó usando cromatografía líquida de alta resolución (HPLC) con detector ultravioleta y empleando extracción en fase sólida (SPE) para la limpieza de las muestras.

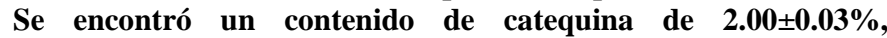
$2.96 \pm 0.02 \%$ y $0.18 \pm 0.03 \%$ en cortezas de $P$. patula, $P$. oocarpa y $E$. grandis respectivamente. Este contenido de catequina indica el gran potencial en aplicaciones industriales y medicinales para un recurso renovable como la corteza, abundante en la región y cuya disposición en campo afecta ambientalmente las zonas forestales.

Palabras clave - Biomasa, HPLC, SPE, polifenoles, taninos condensados.
\end{abstract}

Abstract - The catechin content in barks of the forest species Pinus patula, Pinus oocarpa and Eucaliptus grandis, cultivated in the Department of Cauca-Colombia, was evaluated. The ethanol extracts were obtained by evaluating the best conditions of

Producto derivado del proyecto de investigación "Obtención de productos lignocelulósicos derivados del pino patula, pino oocarpa y eucalipto grandis en bosques cultivados en el departamento del Cauca". Desarrollado en los laboratorios del Grupo de Investigación en Química Analítica Ambiental del Departamento de Química de la Universidad del Cauca.

R. A. Sarria-Villa, Universidad del Cauca, Popayán, Colombia, email: rodrigosv@unicauca.edu.co.

J. A. Gallo, Universidad del Cauca, Popayán, Colombia, email: jagallo@unicauca.edu.co.

R. Benitez, Universidad del Cauca, Popayán, Colombia, email: rbenitez@unicauca.edu.co.

Como citar este artículo: Sarria-Villa R. A., Gallo, J. A., Benitez, R. Extracción de compuestos fenólicos y contenido de catequina en cortezas de tres especies forestales del Cauca-Colombia, Entre Ciencia e Ingeniería, vol. 15, no. 29, pp. 9-27, enero-junio, 2021. DOI: https://doi.org/ $10.31908 / 19098367.2622$

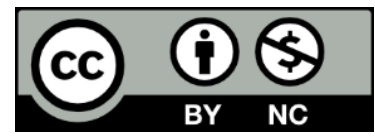

Attribution-NonCommercial 4.0 Intenational (CC By-NC 4.0) particle size, agitation time, solvent-bark ratio and temperature. The catechin content was determined using high performance liquid chromatography (HPLC) with ultraviolet detector and using solid phase extraction (SPE) for sample cleaning. A catechin content of $2.00 \pm 0.03 \%, 2.96 \pm 0.02 \%$ and $0.18 \pm 0.03 \%$ was found in barks of $P$. patula, $P$. oocarpa and $E$. grandis respectively. Catechin content indicates the great potential in industrial and medicinal applications for a renewable resource such as bark, abundant in the region and whose field disposition environmentally affects forest areas.

Keywords - Biomass, HPLC, SPE, polyphenols, condensed tannins.

\section{INTRODUCCIÓN}

$E^{i}$ L Departamento del Cauca es uno de los mayores productores de madera en Colombia, donde las especies de mayor aprovechamiento son el pino y el eucalipto. Del aprovechamiento de la madera se generan grandes volúmenes de corteza, ya que esta representa del 10 al $15 \%$ del peso total del árbol, el cual es un gran recurso que es subutilizado como combustible o quedando esparcido en las plantaciones forestales causando un impacto negativo al medio ambiente [1,2]. La corteza está constituida principalmente por celulosa, lignina y sustancias extraíbles formadas por el metabolismo secundario de las plantas y entre las cuales se encuentran grasas, terpenos, ceras, fenoles, azucares, entre otros [3,4]. Los fenoles son un grupo importante de compuestos presentes en las cortezas, dentro de los cuales vale la pena resaltar a los fenoles simples, ácidos fenólicos, cumarinas, estilbenos, flavonoides, lignanos y taninos [5,6]. Dentro del grupo de los taninos se encuentran los condensados y los hidrolizables, los condensados cuya estructura se basa en la unión de moléculas de catequina en la formación de dímeros, trímeros y tetrámos de gran interés por sus propiedades industriales y biológicas y los taninos hidrolizables formados por moléculas de ácido gálico y cadenas de glucosas $[7,8]$. Los fenoles vegetales se han empleado principalmente en el curtido de pieles y formulación de adhesivos [9,10]; sin embargo, también son empleados en aplicaciones biomédicas. Entre los compuestos fenólicos que se han extraído de la corteza de pino están el 
ácido gálico, ácido ferúlico y ácido p-hidroxibenzoico, los monómeros como la catequina, epicatequina y taxifolin, y flavonoides condensados como las procianidinas. En la Fig. 1 se presenta la estructura química de la catequina.

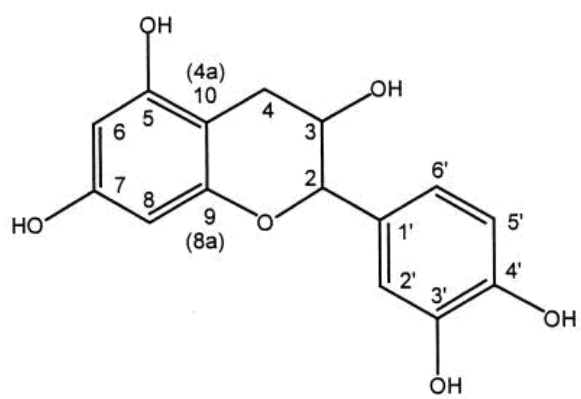

Fig. 1. Estructura química de la catequina.

El contenido de compuestos fenólicos presentes en material vegetal se ha podido determinar gracias al desarrollo de técnicas como la cromatografía de gases (GC), la cromatografía líquida de alta resolución (HPLC), la cromatografía en capa fina (TLC), la espectroscopía infrarroja (IR), espectrometría de masas (MS) y la resonancia magnética nuclear (NMR) [11,12]. De esta manera se han investigado los taninos extraídos de cortezas de especies como Pinus radiata [13], Pinus pinaster y Pinus radiata [14], Pinus pinaster y Pinus massoniana [15], Pinus maritimus [16], Acacia mangium [17] y Acacia confuse [18]. Técnicas como SPE, LLE, SPME y ultrasonido, entre otras, han sido empleadas para el tratamiento de muestras y limpieza de matrices vegetales que contienen polifenoles [19]. La composición de taninos ha sido estudiada en cortezas de Pinus brutia empleando MALDI-TOF-MS y RMN ${ }^{13} \mathrm{C}$ [12]. Es bien conocido que los compuestos fenólicos presentan propiedades antifúngicas, antibióticas, alelopáticas y otros efectos [20,4]. Estos compuestos se han ganado la reputación por su actividad en plantas para el control de ataque de insectos y daños microbianos. Se ha encontrado que compuestos polifenólicos que contienen catequina en su estructura previenen daños cardiovasculares [21,22]. Estudios de la corteza de pino han demostrado su poder antioxidante, anticancerígeno, antimutagénico, antimicrobial y antiinflamatorio [23]. Compuestos fenólicos como la catequina tienen aplicaciones como la prevención de la oxidación de alimentos, astringencia, reacción con metales y proteínas, síntesis de derivados con aplicaciones farmacéuticas, actividad antibacterial y diferentes beneficios medicinales (disminución del colesterol, antialérgico, entre otros). En este trabajo se presenta el estudio de las mejores condiciones de extracción de catequina presente en cortezas de las especies forestales $P$. patula, $P$. oocarpa y E. grandis cultivadas en el Departamento del Cauca-Colombia y la determinación del contenido del polifenol catequina empleando la técnica HPLC con detector ultravioleta visible.

\section{MÉTODOS DE ANÁLISIS}

Muestras de cortezas. Las muestras de corteza de P. oocarpa (18 años) fueron tomadas en la vereda la cabuyerita ubicada al noroccidente de la ciudad de Popayán-Cauca $\left(2^{\circ} 16^{\prime} 36.43^{\prime \prime} \mathrm{N} ; 76^{\circ} 35^{\prime} 42.86^{\prime \prime O}\right)$. Las muestras de corteza de P. patula (18 años) y E. grandis (7 años) se recolectaron en la reserva forestal de Sotará (Paispamba) ubicada al suroccidente de Popayán. En la Fig. 2 se presenta uno de los sitios de muestreo y muestras de cortezas de pino.

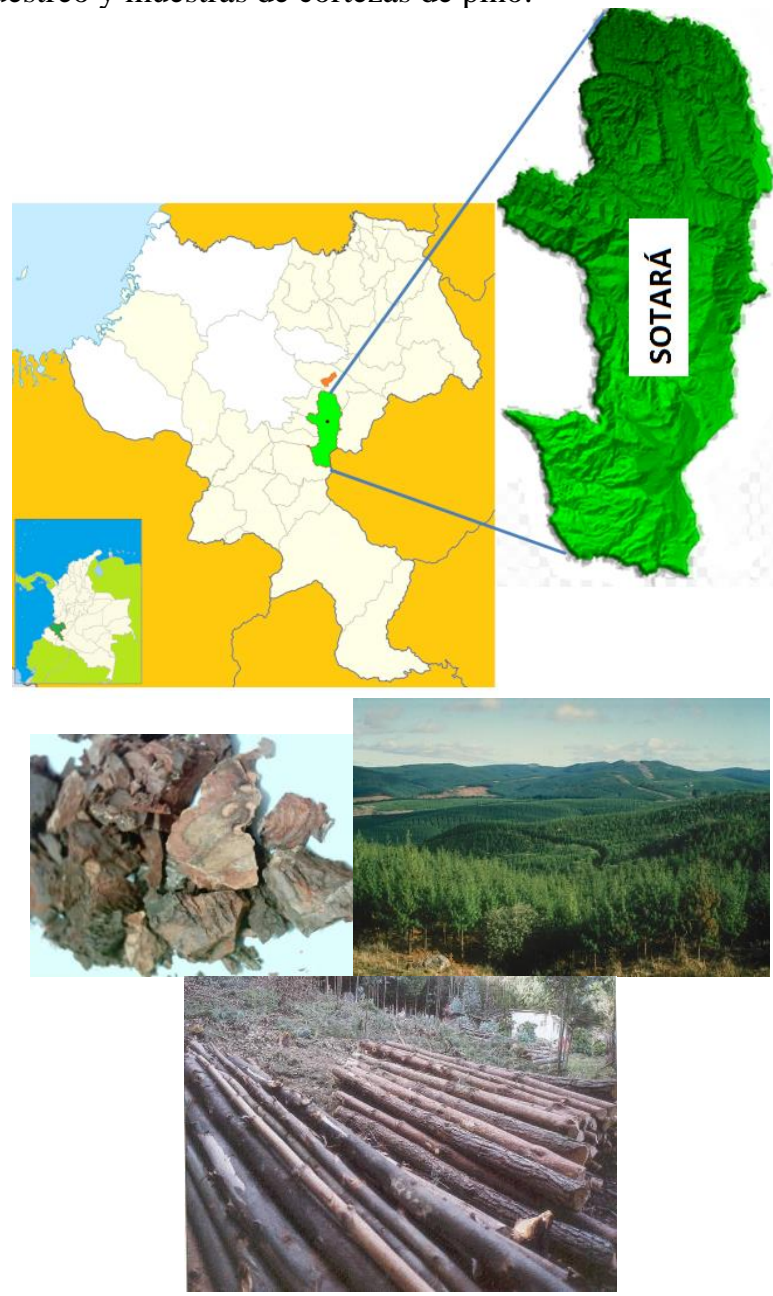

Fig. 2. Zona de muestreo [24] y muestra de corteza de pino Fuente: Grupo GIQA

Molienda de muestras de cortezas. Se tomaron muestras de corteza de 1, 2 y 3 metros de altura de los árboles de las tres especies estudiadas. Las muestras fueron puestas a la sombra para la posterior reducción de tamaño en un molino de aspas de 2 caballos de fuerza y en un molino eléctrico (Retsch SK 100 Estándar Spezst). Las muestras fueron tamizadas entre 1000 y $53 \mu \mathrm{m}$ y almacenadas en bolsas de polietileno evitando humedad e incidencia de la luz.

Análisis proximal de muestras de cortezas. A las muestras de corteza se les determinó porcentaje de humedad, contenido de nitrógeno total, extracto etéreo, cenizas, y contenido de celulosa [25].

Extracción de compuestos fenólicos de muestras de cortezas. Para la obtención de los extractos se emplearon mezclas de etanol-agua, se determinaron las mejores condiciones de extracción como agitación, tiempo, temperatura, relación corteza-solvente y tamaño de partícula. 
Las condiciones de extracción fueron variadas sistemáticamente como se muestra en la tabla I. Se construyó un diseño experimental de 6 factores y tres niveles para cada uno, haciendo una sola réplica para cada experimento. Para tamaño de partícula se evaluaron los tamaños de $1.06,1.70 \mathrm{y}$ $2.36 \mathrm{~mm}$, para temperatura se evaluaron $25^{\circ} \mathrm{C}, 40^{\circ} \mathrm{C}$ y $60^{\circ} \mathrm{C}$, para tiempo se evaluaron 3, 6 y 9 horas, para relación cortezasolvente $(\mathrm{C}: \mathrm{S})$ se evaluaron relaciones $1: 6,1: 8$ y $1: 10$, para composición de solvente se evaluaron cantidades de etanol de $50 \%, 70 \%$ y $100 \%$ completando con agua según la proporción, para revoluciones por minuto se evaluaron 250,380 y 550 rpm. Los extractos se obtuvieron rotaevaporando a una temperatura de $55^{\circ} \mathrm{C}$.

TABLA I.

VARIABLES DE EXTRACCIÓN USADAS PARA CADA EXPERIMENTO

\begin{tabular}{|l|c|c|c|c|c|c|}
\hline Experimento & $(\mathrm{mm})$ & $\left({ }^{\circ} \mathrm{C}\right)$ & $(\mathrm{h})$ & $\mathrm{C}: \mathrm{S}$ & $\mathrm{EtOH}$ & $(\mathrm{rpm})$ \\
\hline & & & & & & \\
$(\mathrm{mm})$ & $1.06-2.36$ & 40 & 6 & $1: 8$ & 70 & 380 \\
\hline$\left({ }^{\circ} \mathrm{C}\right)$ & 1.70 & $25-60$ & 6 & $1: 8$ & 70 & 380 \\
\hline$(\mathrm{h})$ & 1.70 & 40 & $3-9$ & $1: 8$ & 70 & 380 \\
\hline $\mathrm{C}: \mathrm{S}$ & 1.70 & 40 & 6 & $1: 6-1: 10$ & 70 & 380 \\
\hline $\mathrm{EtOH}$ & 1.70 & 40 & 6 & $1: 8$ & $50-100$ & 380 \\
\hline$(\mathrm{rpm})$ & 1.70 & 40 & 6 & $1: 8$ & 70 & $250-550$ \\
\hline
\end{tabular}

Temperatura $\left({ }^{\circ} \mathrm{C}\right)$, Tiempo de extracción (h), Tamaño de partícula $(\mathrm{mm})$, radio cortezasolvente (C:S), Composición del solvente (Etanol: EtOH) and agitación (rpm).

Purificación de extractos de cortezas por SPE. Se empleó la extracción en fase sólida (SPE) para la limpieza de los extractos previo a la medición del contenido de catequina por HPLC. Se tomaron $0.1 \mathrm{~g}$ del extracto de cortezas de P. patula y P. oocarpa y se disolvieron en $10 \mathrm{~mL}$ de metanol para ser pasados a través de cartuchos de $\mathrm{C}_{18}$ (Sep-Pak $\mathrm{C}_{18}$ ), los cuales se acondicionaron con 8 $\mathrm{mL}$ de metanol, $4 \mathrm{~mL}$ de agua a $\mathrm{pH} 7$ y $5 \mathrm{~mL}$ de una solución de $\mathrm{HCl}$ $0.01 \mathrm{M}$, la muestra se cargó y se lavó con $10 \mathrm{~mL}$ de agua a $\mathrm{pH} 7$, finalmente la muestra es eluída con $12 \mathrm{~mL}$ de metanol, esta se lleva a sequedad haciendo pasar nitrógeno por la solución y luego a temperatura controlada de $40{ }^{\circ} \mathrm{C}$, se afora a $5 \mathrm{~mL}$ con la fase móvil y por último se procede con la inyección del filtrado en el cromatógrafo de líquidos [26,27]. Para muestras de extractos de corteza de Eucaliptus grandis se empleó una columna de sílica (40$63 \mu \mathrm{m})$. Inicialmente la sílica se secó en un horno a $150^{\circ} \mathrm{C}$ por cuatro horas, se dejó enfriar y luego se elaboró una columna de aproximadamente $5 \mathrm{~cm}$ de alto por $1 \mathrm{~cm}$ de diámetro. A continuación, se procedió a activar la columna adicionando $5.0 \mathrm{~mL}$ de metanol puro, $3.0 \mathrm{~mL}$ de agua deionizada y $5.0 \mathrm{~mL}$ de una solución acuosa de $\mathrm{HCl} 0.01 \mathrm{M}$. Posteriormente se procedió a adicionar la mezcla a separar. Una vez incluida la mezcla se procedió a adicionar $5 \mathrm{~mL}$ de metanol grado HPLC y por último $5 \mathrm{~mL}$ de una mezcla agua-metanol 50-50. El líquido recogido en el erlenmeyer con desprendimiento se llevó a sequedad en la estufa de aire a $45^{\circ} \mathrm{C}$ y se diluyó en fase móvil para ser filtrado e inyectado en el equipo de cromatografía líquida de alta resolución [27].

Análisis de catequina por HPLC. El análisis de catequina en las cortezas de $P$. patula, $P$. oocarpa y E. grandis se realizó empleando HPLC. Soluciones estándar fueron preparadas a partir de catequina al 98\% marca Sigma-Aldrich. Se empleó un cromatógrafo líquido marca Waters 1515, detector UV-Vis 2487 marca Waters, una columna $\mu$-Bonda Pack $\mathrm{C}_{18}$, temperatura ambiente, elución isocrática a $1.0 \mathrm{~mL} / \mathrm{min}, 15$ minutos de corrida y $10 \mu \mathrm{L}$ de inyección. La fase móvil para el análsis de extractos de corteza de $P$. patula estuvo constituida por $0.5 \%$ de Metanol en Á. Acético 0.01 M-Acetonitrilo $(96.5 \%-3.5 \%)$. La fase móvil empleada para el análisis de extractos de corteza de P. oocarpa constituida por Á. Fosfórico $0.1 \%$ en AguaAcetonitrilo $(88.0 \%-12.0 \%)$ y para el análisis de extractos de $E$. grandis conformada por Á. Fórmico $0.1 \%$ en Agua -Acetonitrilo $(90.0 \%-10.0 \%)$. La detección se realizó a $280 \mathrm{~nm}$ para $P$. patula y $P$. oocarpa y $210 \mathrm{~nm}$ para E. grandis. Todas las muestras fueron filtradas por filtros de membrana de $0.45 \mu \mathrm{m}$ previo al análisis por HPLC [28,29].

Análisis estadístico. Se realizó el análisis de varianza para observar diferencias estadísticamente significativas en las variaciones de condiciones de extracción de compuestos fenólicos. Para este análisis se empleó el programa Statgraphics Centurion. También se realizó análisis de varianza para comparar el contenido de compuestos fenólicos en las tres especies forestales estudiadas empleando el programa SPSS versión 11.5.

\section{RESULTADOS Y DISCUSIÓN}

A las muestras de corteza de las tres especies estudiadas, una vez molidas y tamizadas, se les realizó un análisis proximal para determinar el contenido de humedad, nitrógeno total, extracto etéreo, contenido de lignina y celulosa. En la tabla II se encuentran los porcentajes reportados para las muestras de corteza de $P$. patula, $P$. oocarpa y E. grandis.

formación de complejos, por lo cual la degradación de dichos metales plantea una mayor preocupación [23].

TABLA II

ANÁLISIS PROXIMAL DE LAS MUESTRAS DE CORTEZA DE $P$. PATULA, P. OOCARPA Y E. GRANDIS

\begin{tabular}{cccc}
\hline PROPIEDAD & $\begin{array}{c}\text { Pinus patula } \\
(\%)\end{array}$ & $\begin{array}{c}\text { Pinus oocarpa } \\
(\%)\end{array}$ & $\begin{array}{c}\text { Eucaliptus grandis } \\
(\%)\end{array}$ \\
\hline Humedad & $14.16 \pm 0.03$ & $13.21 \pm 0.03$ & $7.09 \pm 0.05$ \\
Nitrógeno Total & $0.76 \pm 0.04$ & $0.76 \pm 0.02$ & $0.34 \pm 0.03$ \\
Extracto Etéreo & $2.41 \pm 0.05$ & $2.54 \pm 0.02$ & $0.64 \pm 0.02$ \\
Cenizas & $0.83 \pm 0.08$ & $6.79 \pm 0.03$ & $8.73 \pm 0.02$ \\
Celulosa & $27.87 \pm 0.06$ & $24.97 \pm 0.04$ & $32.65 \pm 0.04$ \\
\hline
\end{tabular}

Se puede observar en la tabla II que las muestras de corteza de $P$. patula y $P$. oocarpa presentan una cantidad mayor de nitrógeno total con respecto a las muestras de E. grandis. En cuanto al contenido de grasa (extracto etéreo), las muestras de corteza de $P$. patula y $P$. oocarpa presentaron valores de 2.41 y $2.54 \%$ respectivamente, mientras que las muestras de $E$. grandis solo presentaron un $0.64 \%$. En cuanto al contenido de cenizas el mayor porcentaje lo presentaron las muestras de corteza de E. grandis, seguido de las muestras de corteza de $P$. oocarpa y por ultimo las muestras de $P$. patula. Las muestras de E. grandis presentaron el mayor porcentaje de celulosa, seguidas de las muestras de corteza de $P$. patula y $P$. oocarpa. El contenido de proteína bruta de las especies estudiadas fue similar al de otras especies de pino como $P$. ponderosa $(1.10 \%)$. El contenido de grasa fue muy similar al de especies de $P$. ponderosa $(2.50 \%)$, mientras que el contenido de cenizas estuvo por debajo del que presenta esta especie $(1.10 \%)$. Cortezas con alto contenido de mineral sugieren ser usadas como compost o como sustrato para el crecimiento de plantas 
[30]. Teniendo en cuenta los resultados de análisis proximal de otras especies de pino, contenidos de carbono, oxígeno y nitrógeno (C: 52.40, N: 2.70, O: 38.30) [31,32], indican que estas especies son una importante fuente nutricional para animales a partir de los cuales se puede obtener una fuente carbohidratos. Para la obtención de los extractos de cortezas de $P$. patula, $P$. oocarapa y $E$. grandis se determinaron las mejores condiciones de extracción de acuerdo con la tabla I. En la extracción de taninos a partir de la corteza de E. grandis se pudo observar que los solventes que presentaron mayor porcentaje de extracción fueron las mezclas agua-etanol 50-50 y 30-70 con unos porcentajes muy similares de 9.07 y $9.02 \%$ respectivamente. Desde el punto de vista económico para un aprovechamiento industrial de la corteza de $E$. grandis es mas aconsejable la mezcla agua-etanol (50:50), además el agua es un solvente menos volátil que el etanol, evitando pérdidas durante el proceso de extracción si este se realiza a tanque abierto. Para corteza de $P$. oocarpa la mezcla agua-etanol (3070) permitió extraer taninos en un $6.71 \%$, con mezclas etanolagua (50-50) y etanol $100 \%$ se obtuvieron porcentajes de extracción del 5.70 y $6.80 \%$ respectivamente. Desde el punto de vista industrial la mezcla agua-etanol (30-70) es la más adecuada para extraer taninos a partir de la corteza de $P$. oocarpa. Para el proceso de extracción a partir de la corteza de $P$. patula se pudo observar que empleando etanol $(100 \%)$ se obtiene el mayor porcentaje de extracto total $(9.37 \%)$; sin embargo, la mezcla etanol:agua (70:30) permitió obtener porcentajes de extracto total $(9.14 \%)$, muy cercanos a las obtenidas con etanol $100 \%$, siendo esta última más favorable desde el punto de vista industrial. Con agua-etanol (50:50) y agua $(100 \%)$ se obtuvieron porcentajes de extracto total del 9.07 y $9.19 \%$ respectivamente para corteza de Eucaliptus grandis. Al realizar la prueba ANOVA se observaron diferencias significativas a las tres temperaturas ensayadas para las tres especies forestales con $(\mathrm{Fcal}=19.51>\mathrm{Ftab}=7.71$, $\alpha: 0.05), \quad($ Fcal $=21.21>$ Ftab $=7.71, \quad \alpha: 0.05)$ (Fcal $=19.55>\mathrm{Ftab}=7.71, \alpha: 0.05$ ) para $P$. patula, $P$. oocarpa y $E$. grandis respectivamente. El empleo de metanol y mezclas metanol/agua para extraer fenoles de la corteza de Pinus radiata (agua 100\%, agua:metanol 7:3, agua:metanol 1:1, agua:metanol $3: 7$, metanol $100 \%$ ) ha permitido obtener a partir de esta especie porcentajes promedio de extracción del $8.3,11.98,16.04$ y $21.93 \%$ respectivamente; las extracciones de esta especie de pino chileno se realizaron a muestras de corteza de 27 años, tamaño de partícula pasando la corteza por mallas de 40-60 mesh, agitación de $100 \mathrm{rpm}$ y $70^{\circ} \mathrm{C}$ de calentamiento. Se notó que el incremento de metanol aumenta el porcentaje de extracción [33]. En otros trabajos se ha obtenido un $2.23 \%$ de extracto total para $P$. cooperi y $10.61 \%$ para $P$. ayacahuite con agua $(100 \%)$, mientras que en el extracto etanólico $(50 \%)$ los rendimientos fueron de $6.66 \%$ para $P$. engelmannii a $19.4 \%$ para $P$. ayacahuite; donde se pudo observar que el rendimiento en extracto obtenido con etanol al 50\% fue el doble o mayor al obtenido con agua [34]. Para corteza de E. grandis un nivel de agitación de $250 \mathrm{rpm}$ en adelante mostró porcentajes de extracción constantes $(9.06$, 9.07 y $9.08 \%$ para 250,380 y $550 \mathrm{rpm}$ respectivamente), lo que indica que la corteza no requiere de una agitación muy vigorosa. Para cortezas de $P$. oocarpa se observa que los porcentajes de extracción con agitaciones de $250 \mathrm{rpm}$ y mayores que esta no difieren mucho entre sus valores, pero a una velocidad de agitación de $380 \mathrm{rpm}$ se obtienen los mayores porcentajes de extracción (29.73\%). Si el interés es la disminución del gasto energético, la agitación adecuada es la de $250 \mathrm{rpm}$ (28.76\%). Para cortezas de Pinus patula y Eucalipto grandis a $250 \mathrm{rpm}$ se obtuvieron porcentajes de recuperación de 9.56 y $9.06 \%$ respectivamente. Los resultados de ANOVA indicaron la existencia de diferencias significativas para las tres revoluciones ensayadas en las tres especies forestales con Fcal $>\mathrm{Ftab}, \alpha: 0.05$. La tabla III muestra los resultados obtenidos con el diseño experimental aplicado.

TABLA III

RESULTADOS DEL PROCESO DE EXTRACCIÓN DE COMPUESTOS FENÓLICOS EN CORTEZAS DE P. PATULA, P. OOCARPA Y E. GRANDIS

\begin{tabular}{|c|c|c|c|c|}
\hline & & P. patula & P. оосагра & E. grandis \\
\hline Variable & & & \% Extracto & \\
\hline \multirow[t]{3}{*}{ Tiempo (h) } & 3 & 4.77 & 30.04 & 9.08 \\
\hline & 6 & 5.30 & 28.63 & 9.09 \\
\hline & 9 & 6.02 & 28.36 & 9.10 \\
\hline \multirow{3}{*}{$\begin{array}{l}\text { Tamaño de } \\
\text { partícula }(\mathrm{mm})\end{array}$} & 1.06 & 4.77 & 24.31 & 10.21 \\
\hline & 1.70 & 2.84 & 7.43 & 9.42 \\
\hline & 2.36 & 1.29 & 7.34 & 9.11 \\
\hline \multirow{3}{*}{$\begin{array}{l}\text { Temperatura } \\
\left({ }^{\circ} \mathrm{C}\right)\end{array}$} & 25 & 6.09 & 29.66 & 19.05 \\
\hline & 40 & 9.37 & 30.10 & 19.28 \\
\hline & 60 & 9.45 & 30.26 & 19.76 \\
\hline \multirow{3}{*}{$\begin{array}{l}\text { Relación de } \\
\text { solvente } \\
\text { (etanol:agua) }\end{array}$} & 50 & 8.96 & 5.70 & 9.07 \\
\hline & 70 & 9.14 & 6.71 & 9.02 \\
\hline & 100 & 9.37 & 6.80 & 9.19 \\
\hline \multirow{3}{*}{$\begin{array}{l}\text { Relación } \\
\text { corteza-solvente } \\
\text { (C:S) }\end{array}$} & $1-6$ & 3.92 & 25.09 & 7.11 \\
\hline & $1-8$ & 4.28 & 25.42 & 8.24 \\
\hline & $1-10$ & 4.77 & 25.75 & 8.88 \\
\hline \multirow{3}{*}{$\begin{array}{l}\text { Velocidad de } \\
\text { agitación (rpm) }\end{array}$} & 250 & 9.56 & 28.76 & 9.06 \\
\hline & 380 & 10.19 & 29.73 & 9.07 \\
\hline & 550 & 9.98 & 28.42 & 9.08 \\
\hline
\end{tabular}

El tiempo adecuado para la obtención de extractos de corteza de $P$. patula fue de 9 horas con un $6.02 \%$ de extracto total. Con tiempos de 3 y 6 horas se obtuvieron 4.77 y $5.30 \%$ de extracto total respectivamente. Tiempos de extracción superiores a 6 horas incrementan los costos de extracción. En extractos de corteza de $P$. pinaster se ha encontrado que tiempos prolongados de extracción no son necesarios para la liberación de compuestos fenólicos de la estructura de cortezas de pino [35]. Según los valores encontrados el tiempo adecuado para alcanzar porcentajes de extracción adecuados están por encima de 3 horas, encontrándose los mejores 
porcentajes de extracción entre 3 y 9 horas. Se encontraron los mejores porcentajes de extracción de $6.02 \%(9 \mathrm{~h}), 28.63 \%(6 \mathrm{~h})$ y $9.10 \%(9 \mathrm{~h})$ para cortezas de $P$. patula, P. oocarpa y E. grandis respectivamente. Tiempos de extracción superiores a 3 horas incrementan costos de extracción. La prueba ANOVA para observar diferencias en cada uno de los tratamientos arrojó la no existencia de diferencias en la variación del tiempo de extracción para la corteza de $P$. patula (Fcal $=0.12<\mathrm{Ftab}=7.71, \alpha: 0.05)$ y $E$. grandis $\quad(\mathrm{Fcal}<\mathrm{Ftab}$, $\alpha: 0.05)$. Se observaron diferencias al variar el tiempo en la extracción de compuestos fenólicos de la corteza de $P$. oocarpa (Fcal $>$ Ftab, $\alpha: 0.05$ ). Trabajos hechos en Cuba, han reportado rendimientos de obtención de extracto de 8.29 y $10.19 \%$ para $P$. caribea y $P$. cubensis respectivamente a tiempos inferiores de extracción de 3h [36,37]. La relación de corteza de $P$. patula-solvente de 1:10 permitió obtener el mayor porcentaje de extracto total del $4.77 \%$. Con relaciones de corteza-solvente de 1:6 y 1:8 se obtuvieron porcentajes de extracto total del 3.92 y $4.28 \%$ respectivamente. Relaciones de corteza-solvente de 1:10 permitieron obtener los mayores porcentajes de extracción de $25.75 \%$ y $8.88 \%$ para cortezas de $P$. oocarpa y $E$. grandis respectivamente. El empleo de un tamaño de partícula menor $(1.06 \mathrm{~mm})$ permitió obtener el mayor porcentaje de extracto total para la especie de $P$. patula $(4.77 \%)$. Se observa que, al disminuir el tamaño de partícula, aumenta el porcentaje de extracto total debido a la mayor superficie de contacto entre la muestra y el solvente. Para cortezas de $P$. oocarpa y E. grandis también se obtuvieron los mayores porcentajes de extracción al menor tamaño de partícula $(1.06 \mathrm{~mm})$ de $24.31 \%$ y $10.21 \%$ respectivamente. $\mathrm{La}$ prueba de ANOVA arrojó diferencias significativas en el tamaño de partícula para la extracción de $P$. patula y $P$. oocarpa con (Fcal $>$ Ftab, a:0.05). Para la extracción de $E$. grandis no se observaron diferencias significativas para el tamaño (Fcal $=0.004<\mathrm{Ftab}=7.71, \alpha: 0.05)$.

La temperatura a la que se logró mayor extracción de compuestos fenólicos a partir de la corteza de $P$. patula fue la de $60^{\circ} \mathrm{C}$ con un porcentaje de extracto total del $9.45 \%$, superior al obtenido a temperatura de $25^{\circ} \mathrm{C}$ y $40^{\circ} \mathrm{C}$ de $6.09 \%$ y $9.37 \%$ respectivamente. Para cortezas de $P$. oocarpa y $E$. grandis la temperatura más alta de $60^{\circ} \mathrm{C}$ permitió obtener los porcentajes de extracción más altos de $30.26 \%$ y $19.76 \%$ respectivamente. Se observó efecto estadísticamente significativo por temperatura para la extracción de compuestos fenólicos de la corteza de $P$. patula (Fcal $>$ Ftab, $\alpha: 0.05$ ). No se observó efecto estadísticamente significativo por temperatura para la extracción en cortezas de $P$. oocarpa y E. grandis (Fcal $<$ Ftab, $\alpha: 0.05)$. El análisis de varianza con respecto al porcentaje de extracto fenólico de corteza de $P$. patula permitió observar que no existen efectos estadísticamente significativos al variar las condiciones de cada una de las variables estudiadas (A:Tiempo, B:Tamaño de partícula, C:Temperatura, D:Etanol, E:Relación C:S, F:Revoluciones). Solamente se observó un efecto estadísticamente significativo para $(\mathrm{DxF})$ con un $\mathrm{p}<0.05$ para la extracción de corteza de $P$. patula, indicando su efecto negativo, al no necesitarse la cantidad de etanol ni revoluciones de agitación más altas para una extracción efectiva. Para el análisis de varianza de la extracción de compuestos fenólicos de la corteza de $P$. oocarpa no presentó efectos estadísticamente significativos $(\mathrm{p}>0.05)$ para ninguna de las variables en estudio (A:Tiempo, B:Tamaño de partícula, C:Temperatura, D:Etanol, E:Relación $\mathrm{C}: \mathrm{S}, \mathrm{F}:$ Revoluciones). Las variables que marcaron mayor importancia fueron un efecto negativo para $(\mathrm{FxE})$ y efecto positivo (BxE), indicando que efectos de relación C:S y agitación a la baja pueden incrementar el porcentaje de extracción y efectos tamaño de partícula y relación C:S altos pueden incrementar el porcentaje de extracción de compuestos fenólicos. El análisis de varianza para la extracción de compuestos fenólicos de cortezas de E. grandis no mostró efecto estadísticamente significativo para ninguna de las variables en estudio (A:Tiempo, B:Tamaño de partícula, C:Temperatura, D:Etanol, E:Relación C:S, F:Revoluciones) con $\mathrm{p}>0.05$. Las variables conjuntas de mayor efecto fueron (AxE) con efecto negativo y (AxC) con efecto positivo, lo que indica que tiempos y relaciones $\mathrm{C}: \mathrm{S}$ no muy altos permiten buenos porcentajes de extracción y el aumento del tiempo y temperatura incrementan la extracción. En la tabla IV se presenta el análisis de varianza en la variación de condiciones para la obtención de extracto fenólico para las tres especies en estudio.

TABLA IV

ANÁLISIS DE VARIANZA PARA OBTENCIÓN DE EXTRACTO FENÓLICO DE CORTEZAS DE P. PATULA, P. OOCARPA Y E. GRANDIS

\begin{tabular}{|c|c|c|c|c|c|c|c|}
\hline \multirow[t]{2}{*}{ Fuente } & \multirow[t]{2}{*}{$G l$} & \multicolumn{3}{|c|}{ Razón-F } & \multicolumn{3}{|c|}{ Valor-P } \\
\hline & & $\begin{array}{c}P . \\
\text { patula }\end{array}$ & $\begin{array}{c}P . \\
\text { oocarpa }\end{array}$ & $\begin{array}{c}\text { E. } \\
\text { grandis }\end{array}$ & $\begin{array}{c}P . \\
\text { patula }\end{array}$ & $\begin{array}{c}P . \\
\text { oocarpa }\end{array}$ & $\begin{array}{c}E . \\
\text { grandis }\end{array}$ \\
\hline A:Tiempo & 1 & 1.85 & 0.22 & 0.04 & 0.19 & 0.64 & 0.84 \\
\hline $\begin{array}{l}\text { B:Tamaño } \\
\text { partícula }\end{array}$ & 1 & 2.30 & 0.46 & 0.00 & 0.15 & 0.50 & 0.99 \\
\hline C:Temperatura & 1 & 0.34 & 0.52 & 1.40 & 0.57 & 0.48 & 0.25 \\
\hline D:Etanol & 1 & 0.12 & 0.52 & 0.73 & 0.73 & 0.48 & 0.40 \\
\hline E:Relación C:S & 1 & 1.01 & 0.33 & 0.45 & 0.39 & 0.57 & 0.51 \\
\hline F:Revoluciones & 1 & 2.28 & 0.01 & 0.60 & 0.15 & 0.94 & 0.45 \\
\hline AA & 1 & 0.17 & 0,00 & 1.37 & 0.69 & 0.98 & 0.26 \\
\hline $\mathrm{AB}$ & 1 & 0.11 & 0.40 & 0,00 & 0.74 & 0.53 & 0.98 \\
\hline $\mathrm{AC}$ & 1 & 0.09 & 0,00 & 2.15 & 0.77 & 0.96 & 0.16 \\
\hline $\mathrm{AD}$ & 1 & 0.14 & 1.22 & 0.00 & 0.72 & 0.28 & 0.99 \\
\hline $\mathrm{AE}$ & 1 & 0.05 & 0.80 & 0.64 & 0.82 & 0.38 & 0.43 \\
\hline $\mathrm{AF}$ & 1 & 0.01 & 0.66 & 2.23 & 0.92 & 0.43 & 0.15 \\
\hline BB & 1 & 0.07 & 0.43 & 0.05 & 0.79 & 0.52 & 0.83 \\
\hline $\mathrm{BC}$ & 1 & 0.15 & 0.21 & 0.67 & 0.70 & 0.66 & 0.42 \\
\hline $\mathrm{BD}$ & 1 & 0.62 & 0,00 & 0.47 & 0.44 & 0.97 & 0.50 \\
\hline $\mathrm{BE}$ & 1 & 0.11 & 1.96 & 0.01 & 0.75 & 0.18 & 0.93 \\
\hline $\mathrm{BF}$ & 1 & 0,00 & 2.40 & 0.40 & 0.97 & 0.14 & 0.54 \\
\hline $\mathrm{CC}$ & 1 & 3.09 & 0.22 & 0.00 & 0.10 & 0.64 & 0.99 \\
\hline $\mathrm{CD}$ & 1 & 1.22 & 0.41 & 0.68 & 0.28 & 0.53 & 0.42 \\
\hline $\mathrm{CE}$ & 1 & 0.97 & 0.95 & 0.00 & 0.34 & 0.34 & 0.99 \\
\hline $\mathrm{CF}$ & 1 & 0.47 & 0.67 & 0.44 & 0.50 & 0.42 & 0.52 \\
\hline DD & 1 & 0.09 & 0.52 & 0.81 & 0.76 & 0.48 & 0.39 \\
\hline $\mathrm{DE}$ & 1 & 0.00 & 0.06 & 0.02 & 0.98 & 0.81 & 0.88 \\
\hline DF & 1 & 4.57 & 1.66 & 0.63 & 0.05 & 0.21 & 0.44 \\
\hline $\mathrm{EE}$ & 1 & 0.43 & 0.74 & 0.00 & 0.52 & 0.40 & 0.99 \\
\hline $\mathrm{EF}$ & 1 & 2.33 & 1.20 & 0.04 & 0.14 & 0.29 & 0.85 \\
\hline FF & 1 & 0.36 & 3.39 & 1.37 & 0.56 & 0.08 & 0.26 \\
\hline Error total & 18 & & & & & & \\
\hline Total (corr.) & 45 & & & & & & \\
\hline
\end{tabular}

R-cuadrada $=58,7487$ porciento, R-cuadrada (ajustada por g.l.) $=0,0$ porciento, Error estándar del est. $=2,80701$, Error absoluto medio $=1,37271$, Estadístico Durbin-Watson $=1,41427(\mathrm{P}=0,0125)$, Autocorrelación residual de Lag $1=0,257528$

El análisis estadístico y el resultado en los porcentajes de extracción permitieron determinar las mejores condiciones de 
extracción de compuestos fenólicos para cortezas de $P$. patula, $P$. oocarpa y E. grandis, los cuales se presentan en la tabla V. Se debe tener en cuenta que el $P$. oocarpa es más voluminoso que el $P$. patula, esto indica que la corteza del $P$. oocarpa es más abundante y a la vez debe estar relacionado con el contenido de compuestos fenólicos. Tanto las muestras de cortezas de $P$. patula y $P$. oocarpa fueron tomadas de árboles de 18 años, edad en la cual el árbol ya puede ser cosechado. Este tipo de pinos pueden ser cosechados entre 15 y 25 años. Las muestras de cortezas de E. grandis fueron tomadas de árboles de 7 años, este tipo de árboles maduran más pronto que los pinos y pueden ser cosechados desde los 7 años, lo que a su vez puede estar relacionado con su contenido de compuestos fenólicos en la corteza. Cabe mencionar que el crecimiento de estas especies forestales depende de variables como altura, temperatura, tipo de suelo, $\mathrm{pH}$, agua, entre otras [38].

TABLA V

PARÁMETROS ÓPTIMOS DE EXTRACCIÓN DE TANINOS A PARTIR DE CORTEZA DE P. PATULA, P. OOCARPA Y E. GRANDIS

\begin{tabular}{|c|c|c|c|}
\hline Parámetro & $\begin{array}{l}\text { Corteza } P \text {. } \\
\text { patula }\end{array}$ & $\begin{array}{c}\text { Corteza } P . \\
\text { oocarpa }\end{array}$ & $\begin{array}{c}\text { Corteza } E \text {. } \\
\text { grandis }\end{array}$ \\
\hline Tiempo (h) & 3 & 3 & 3 \\
\hline $\begin{array}{l}\text { Tamaño de partícula } \\
(\mathrm{mm})\end{array}$ & 1.06 & 1.06 & 1.06 \\
\hline Temperatura $\left({ }^{\circ} \mathrm{C}\right)$ & 60 & 60 & 60 \\
\hline $\begin{array}{l}\text { Relación de solvente } \\
\text { (agua: etanol)\% }\end{array}$ & 30: 70 & $30: 70$ & 50: 50 \\
\hline $\begin{array}{l}\text { Relación corteza- } \\
\text { solvente }(\mathrm{g} / \mathrm{mL})\end{array}$ & $1-10$ & $1-10$ & $1-10$ \\
\hline $\begin{array}{l}\text { Velocidad de agitación } \\
(\mathrm{rpm})\end{array}$ & 250 & 380 & 250 \\
\hline
\end{tabular}

Las mejores condiciones de extracción permitieron obtener porcentajes de extracto de compuestos fenólicos del $10.90 \pm 0.06,29.67 \pm 0.05$ y $19.92 \pm 0.07 \%$ para corteza de $P$. patula, $P$. oocarpa y $E$ grandis respectivamente. Una vez fijadas las condiciones para la extracción de polifenoles a partir de las cortezas, las muestras fueron filtradas y se concentraron a presión reducida en rotavapor. Se tomaron 0.5 $\mathrm{g}$ del extracto obtenido y se disolvieron en etanol. Las muestras fueron sometidas a un proceso de limpieza de matriz usando SPE [26,39]. La estandarización del proceso de SPE permitió obtener porcentajes de recuperación para catequina del $98 \%, 97 \%$ y $96 \%$ para extractos de corteza de $P$. patula, $P$. oocarpa y E. grandis respectivamente. El uso de la SPE ha permitido la extracción de compuestos bioactivos como catequina, quercetina y (E)-Resverattrol presentes en vino rojo, alcanzando porcentajes de recuperación del $99 \%$ para (E)-Resverattrol [40]. La determinación de polifenoles en material vegetal por espectrometría de masas con ionización por electrospray (ESI-MS) con limpieza de muestras usando SPE ha permitido porcentajes de recuperación entre el 69 y el $91 \%$ [41]. Una vez realizado el proceso SPE, el eluato fue concentrado a sequedad con corriente de nitrógeno y redisuelto en $5 \mathrm{~mL}$ de fase móvil y pasadas por filtros de membrana de $0.45 \mu \mathrm{m}$ para ser analizadas por HPLC. En la
Fig. 3 se presentan los cromatogramas de las muestras de extractos de cortezas de $P$. patula, $P$. oocarpa y E. grandis [28]. Se puede observar como la SPE permite eliminar posibles interferencias y se observa un pico resuelto y representativo para la catequina como analito de interés.
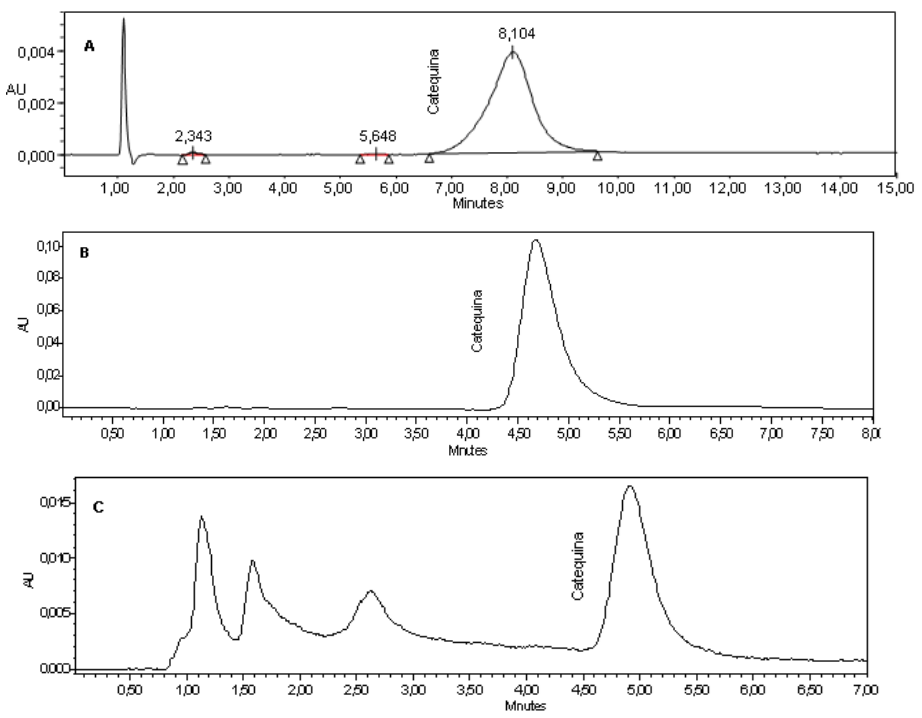

Fig. 3. Cromatograma de catequina para A) corteza de $P$. patula, B) corteza de $P$. oocarpa, C) corteza de E. grandis

El método cromatográfico mostró buena señal de la catequina, los picos cromatográficos de la catequina para las tres especies de pino mostraron buena simetría y resolución. Se presentaron tiempos de retención para catequina de $8.05 \pm 0.03 \mathrm{~min}, 4.72 \pm 0.06 \mathrm{~min}$ y $4.93 \pm 0.05 \mathrm{~min}$ para extractos de corteza de $P$. patula, $P$. oocarpa y $E$. grandis respectivamente. Se observa que la variación de la composición de la fase móvil permite separar y cuantificar de forma adecuada la catequina, teniendo en cuenta que el método cromatográfico se construyó con el estándar de catequina. La fase móvil compuesta de un solvente orgánico y un pequeño porcentaje de ácido permite separar de forma adecuada compuestos fenólicos presentes en matrices vegetales [42]. La variación de la fase móvil permitió comprender las interacciones de la catequina con la fase móvil y la fase estacionaria, se pudo observar con la fase móvil empleada para separar la catequina presente en la corteza de $P$. patula $(0.5 \% \mathrm{MeOH}$ en A. acético 0.01 M-Acetonitrilo: 96.5\%-3.5\%) un mayor tiempo de retención (8.05 $\mathrm{min}$ ), indicando la influencia de la presencia del metanol en la fase móvil mezclado con ácido acético y acetonitrilo, lo que pudo generar mayor interacción fase móvil-analito [48], esta interacción genera ensanchamiento del pico debido al mayor tiempo que pasa el analito en la columna. Para la separación de catequina en los extractos de $P$. oocarpa y E. grandis donde se usó fase móvil con ácido fosfórico-Acetonitrilo y A. fórmico-Acetonitrilo respectivamente se obtuvieron tiempos de retención similares (4.72 min $P$. oocarpa; $4.93 \mathrm{~min} E$. grandis) con una forma del pico similar y mayor simetría que 
la obtenida para la separación en el extracto de $P$. patula. El empleo de una columna C18 permitió la adecuada separación de la catequina presente en los extractos etanólicos de corteza previa limpieza del extracto empleando SPE conformado por adsorbente de C-18. Entre los factores que más influyen en la retención están la estereoquímica, sustitución en el anillo fenólico, peso molecular y polaridad total [43,12]. Las longitudes de onda de 210 y $280 \mathrm{~nm}$ permitieron detectar el compuesto de interés teniendo en cuenta que la catequina presenta dobles enlaces y átomos de oxígeno con electrones libres facilitando las transiciones $\mathrm{n} \rightarrow \pi^{*} \mathrm{y} \pi \rightarrow \pi^{*}$. El espectro UV-Vis de compuestos fenólicos normalmente presenta bandas de máxima absorbancia entre los 200 y los $300 \mathrm{~nm}$, dependiendo de los sustituyentes del anillo aromático. La absorbancia máxima muchas veces depende de la conjugación, la posición y el número de sustituyentes en el anillo aromático [43,12].

El método para determinar catequina en muestras de corteza de $P$. patula presentó buena linealidad en el rango de 10 a 100 ppm $(\mathrm{r} 2=0.9954)$, un límite de detección de 0.058 ppm y un límite de cuantificación de $0.19 \mathrm{mgL}^{-1}$, el método presentó buena repetitividad en el rango de concentraciones examinado con valores de \%RSD entre 0.197 y 4.532 , valores que no sobrepasan el 5\%, criterio de aceptación para el análisis de catequina [42,23]. El método presentó reproducibilidad con valores de \%RSD por debajo del 5\% (0.063-1.360\%). El método presentó exactitud con un tobt de 1.430, inferior al ttab de 2.31 para ocho (8) grados de libertad y un $95 \%$ de confianza. El método cromatográfico para determinar catequina en muestras de corteza de $P$. oocarpa presentó buena linealidad en el rango de 10 a $20 \mathrm{mgL}^{-1}(\mathrm{r} 2=$ 0.9995), presentó un límite de detección de $0.785 \mathrm{mgL}^{-1} \mathrm{y}$ un límite de cuantificación de $1.837 \mathrm{mgL}^{-1}$, el método presentó buena repetitividad en el rango de concentraciones examinado con valores de \%RSD entre 0.500 y 2.570 , valores que no sobrepasan el 5\%, criterio de aceptación para el análisis de catequina. El método es reproducible porque los valores de

\%RSD están por debajo del 5\% (1.34-1.61). El método es exacto al presentar un tobt de 1.640, inferior al tab de 2.31 para ocho (8) grados de libertad y un $95 \%$ de confianza. El método cromatográfico para determinar catequina en muestras de corteza de E. grandis presentó buena linealidad en el rango de 1 a $100 \mathrm{mgL}^{-1}(\mathrm{r} 2=0.9998)$, presentó un límite de detección de $0.013 \mathrm{mgL}^{-1}$ y un límite de cuantificación de $0.021 \mathrm{mgL}^{-1}$, el método presentó buena repetitividad en el rango de concentraciones examinado con valores de \%RSD entre 0.235 y 3.681 , valores que no sobrepasan el $5 \%$, criterio de aceptación para el análisis de catequina. El método es reproducible porque los valores de \%RSD están por debajo del $5 \%(0.647-1.838 \%)$. El método es exacto al presentar un tobt de 1.941 inferior al ttab de 2.31 para ocho (8) grados de libertad y un $95 \%$ de confianza. Los métodos cromatográficos permitieron cuantificar catequina en muestras de corteza de $P$. patula, $P$. oocarpa y $E$. grandis. Los resultados se encuentran en la Fig. 4. Las muestras de cortezas de $P$. patula presentaron un porcentaje de catequina de $2.00 \pm 0.03 \%$, las muestras de corteza de $P$. oocarpa presentaron un $2.96 \pm 0.02 \%$ de catequina y $E$. grandis un $0.18 \pm 0.03 \%$. Especies como la $P$. marítima presenta contenidos de catequina del $18.9 \%$ [44]. Se han reportado contenidos de catequina del $7 \%$ extraída con acetato de etilo de cortezas de $P$. brutia [44]. También, se han encontrado contenidos de catequina del $8.1 \%$ en cortezas de $P$. pinaster [45]. Contenidos de los dímeros epicatequina y catequina del $40.9 \%$ han sido reportados para cortezas de $P$. marítima, lo que indica que bajo procesos adecuados de hidrólisis se pueden obtener mayores contenidos de catequina en las muestras de cortezas [46].

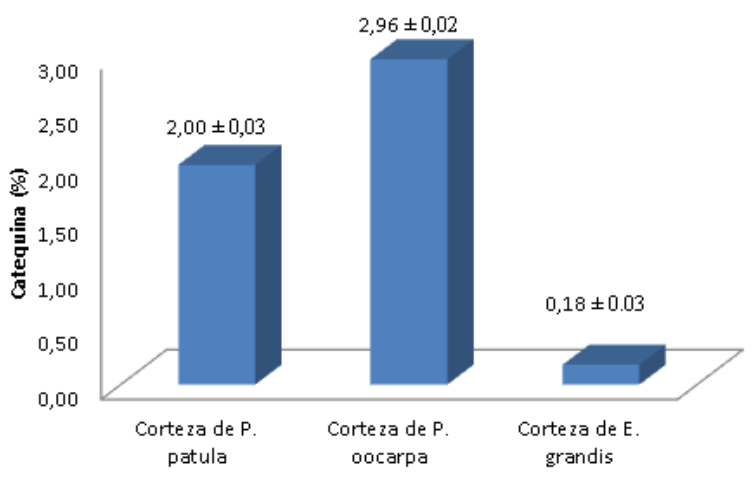

Fig. 4. Contenido de catequina en muestras de cortezas de $P$. patula, $P$. oocarpa y E. grandis.

La prueba ANOVA mostró que existe diferencia entre los contenidos de catequina en corteza de las tres especies forestales estudiadas, presentando un $\mathrm{F}_{\text {calculado }}$ (9968.67) mayor que el $\mathrm{F}_{\text {tabla }}(5.14)$ a un $95 \%$ de confianza. La prueba de tukey permitió observar que existe diferencia honestamente significativa (HSD) entre los contenidos de catequina en cortezas de $P$. patula, $P$. oocarpa con respecto a la de $E$. grandis. La prueba de Tukey indica que no existe diferencia significativa entre el contenido de catequina en las cortezas de $P$. patula y $P$. oocarpa. La caracterización de este tipo de materiales lignocelulósicos permite contar con metodologías para analizar muestras similares. La determinación de catequina en cortezas permite conocer su proporción, respaldar metodologías de aislamiento, síntesis y cuantificación cromatográfica, como también el desarrollo de ensayos biológicos para determinar sus propiedades antibacteriales, antiinflamatorias entre otras [47].

\section{CONCLUSIONES}

Las condiciones adecuadas para el proceso de extracción de compuestos fenólicos a partir de la corteza de P. patula, P. oocarpa y E. grandis a nivel del laboratorio, en cuanto a tamaño de partícula, relación corteza - solvente, agitación, tiempo de extracción, tipo de solvente usado y temperatura fueron establecidas de acuerdo con los resultados obtenidos. Con las cuales se obtuvo un rendimiento de $10.90 \pm 0.06$, $29.67 \pm 0.05$ y $19.92 \pm 0.07 \%$ en peso respectivamente. La cromatografía líquida de alta resolución (HPLC) es una buena 
técnica analítica para la determinación cualitativa y cuantitativa de flavonoides presentes en muestras de corteza, suministrando picos cromatográficos definidos, con bajos límites de detección y cuantificación. Además, los métodos presentaron apropiada linealidad, reproducibilidad, exactitud y precisión. Los métodos permitieron determinar la cantidad de catequina en las muestras. La extracción en fase sólida (SPE) es un excelente método para evitar el efecto de matriz en el análisis de compuestos como la catequina. Los contenidos de catequina en las muestras de corteza de P. patula, P. oocarpa y E. grandis fueron $2.00,2.96$ y $0.18 \%$ respectivamente. La determinación del contenido de catequina, en las tres especies forestales estudiadas con los métodos propuestos indican su viabilidad y buena proyección para su uso.

\section{AGRADECIMIENTOS}

Los autores agradecen a Colciencias, Smurfit Cartón de Colombia, Cootraforc y al Departamento de Química de la Universidad del Cauca por el apoyo brindado durante el desarrollo de esta investigación.

\section{REFERENCIAS}

[1] H. Kofujita, K. Ettyu y M. Ota, "Characterization of the major components in bark from five Japanese tree species for chemical utilization", Wod science and Technology., vol. 33, pp. 223-228, 1999.

[2] C.S. Tavares et al., "Bioproducts from forest biomass: Essential oils and hydrolates from wastes of Cupressus lusitanica Mill. and Cistus ladanifer L”, Industrial Crops and Products., vol. 144, 112034, 2020.

[3] D. Fengel, and G. Wegener, "Wood, chemistry, ultrastructure, reactions". Walter de Gruyter Ed. Nueva York, pp. 90-115, 1989.

[4] I. Mármol et al., "A systematic review of the potential uses of pine bark in food industry and health care", Trends in Food Science \& Technology., vol. 88, pp. 558-566, 2019.

[5] P.M. Dey and J.B. Harborne, "Methods in plant biochemistry. Plant phenolics". Academic Press., vol.1, 552 p, 1989.

[6] P.S. Chiang et al., "Extracting antioxidant phenolic compounds from compressional-puffing pretreated Pinus morrisonicola: Effects of operational parameters, kinetics and characterization", Journal of the Taiwan Institute of Chemical Engineers., vol. 75, pp. 70-76, 2017.

[7] Z. Peng et al., "Quantitative Analysis of Polymeric Procyanidins (Tannins) from Grape (Vitis vinifera) Seeds by Reverse Phase HighPerformance Liquid Chromatography", J. Agric. Food Chem., vol. 49, pp. 26-31, 2001.

[8] N.A. Rosdiana at al., "Characterization of bark extractives of different industrial Indonesian wood species for potential valorization", Industrial Crops and Products, vol. 108, pp. 121-127, 2017.

[9] G.J. Garro, B. Riedl and A. Conner, "Analytical studies on tara tannins". Holzforschung., vol. 51(3), pp. 235-243, 1997.

[10] C.R. China et al., "Suitability of selected vegetable tannins traditionally used in leather making in Tanzania", Journal of Cleaner Production., vol. 251, 119687, 2020.

[11] G.J Soleas, E.P. Diamaudis and D.M. Goldberg, D.M. "Resveratrol: a molecule whose time has come and gone?", J Clin Lab Anal., vol. 11, pp. 287-313, 1997.

[12] M.B. Ucar, G. Ucar, A. Pizzi, O. Gonultasa, "Characterization of Pinus brutia bark tannin by MALDI-TOF MS and 13C NMR"., Industrial Crops and Products., vol. 49, pp. 697-704, 2013.

[13] C.S. Ku, and S.P. Mun, "Characterization of proanthocyanidin in hot water extract isolated from Pinus radiata bark", Wood Sci. Technol, vol. 41, pp. 235-247, 2007.

[14] M. Jerez, M. Pinelo, J. Sineiro, M.J. Núñez, M.J. "Influence of extraction conditions on phenolic yields from pine bark: assessment of procyanidins polymerization degree by thiolysis", Food Chemistry., vol. 94, 406-414, 2006.
[15] H. Weber et al., "Comparison of proanthocyanidins in comercial antioxidants: grape seed and pine bark extracts", J. Agric. Food Chem., vol. 55, pp. 148-156, 2007.

[16] P. Navarrete et al., "MALDI-TOF and 13C NMR characterization of maritime pine industrial tannin extract", Ind. Crops Prod, vol. 32, pp. 105-110, 2010.

[17] Y.B. Hoong et al., "Characterization of Acacia mangium polyflavonoid tannins by MALDI-TOF mass spectrometry and CP-MAS 13C NMR", Eur. Polym. J., vol. 46, pp. 1268-1277, 2010.

[18] Y. Wei et al., 2011. "Qualitative and Quantitative Evaluation of Phenolic Compounds in Iris dichotoma Pall, Phytochemical Analysis., vil. 23(3), pp. 197-207, 2012.

[19] S. Bianchi et al., "Characterization of condensed tannins and carbohydrates in hot water bark extracts of European softwood species", Phytochemistry., vol. 120, pp. 53-61, 2015.

[20] Y. Sattler, C. Grone, A. Zurk, "New compounds of the manumycin group of antibiotics and a fracilitated rouce for their structure elucidation", Org. Chem., vol. 58, 6583, 1993.

[21] P.C. Hollman, M.G. Hertog, M.B. Katan, "Analysis and health effects of flavonoids", Food Chem., vol. 57, pp. 43-6, 1996.

[22] K.A. Cooper, M. Chopra, D.I. Thurnham, "Wine polyphenols and promotion of cardiac health", Nutrition Research Rewiews., vol. 17, pp. 111-129, 2004.

[23] Y-Y. Li, et al., "Effects of Pinus massoniana bark extract on the invasion capability of HeLa cells", Journal of Functional Foods., vol. 24, pp. $520-526,2016$.

[24] Sotará-Cauca.En: https://es.wikipedia.org/wiki/Sotar\%C3\%A1_(Cauca). Consultada: 01/10/2019.

[25] Anual Book of ASTM standars. Vol. 04.09 Feb.1985.

[26] I. Spranger, et al., "Separation of grape and wine proanthocyanidins according to their degree of polymerization", J. Agric. Food Chem., vol. 46, pp. 1390-1396, 1998.

[27] L. Chupin et al., "Characterisation of maritime pine (Pinus pinaster) bark tannins extracted under different conditions by spectroscopic methods, FTIR and HPLC", Ind. Crops Prod., vol. 49, pp. 897-903, 2013.

[28] R. Govindarajan, D.P. Singh, A.K.S. Rawat, "High perfomance liquid chromatography method for quantification of phenolics in Chyavanprash a potent Ayurvedic drug", Journal of Pharmaceutical and Biomedical Analysis., vol. 43, pp. 527-532, 2007.

[29] T.G. Gini, and G.J. Jothi, "Column chromatography and HPLC analysis of phenolic compounds in the fractions of Salvinia molesta Mitchell", Egyptian Journal of Basic and Applied Sciences, vol. 5(3), pp. 197-203, 2018.

[30] G. Ganjeguntea et al., "Decomposition and nutrient release from radiata pine (Pinus radiata) coarse woody debris", Forest Ecology and Management., vol. 187, pp. 197-211, 2004.

[31] L.K. Ernst, "Productos forrajeros a partir de los residuos del bosque". Edit. Lesnaya Prom. Moscú, 166 p, 1982.

[32] F. Herráez, F y J. Gutiérrez, "Caracterización de la corteza de Pinus pinaster Ait. Generada en ls aserraderos de la provincia de Ávila para su uso energético como biomasa”. Universidad Católica de Ávila, 2012. Consultada: 12/01/2020. Disponible en: https://www.interempresas.net/Madera/Articulos/103297-

Caracterizacion-corteza-Pinus-pinaster-Ait-generada-aserraderosprovincia-Avila-uso.html.

[33] D. Hon and N. Shiraishi, "Wood and cellulosic chemistry". Second edition. Marcel Dekker, Inc. New York. 914p, 2000.

[34] M. Castro Y R. Gonzáles, "Comparación del contenido de compuestos fenólicos en la corteza de ocho especies de pino", Madera y Bosques, vol. 9(2), pp. 41-49, 2003.

[35] M. Jerez et al., "Influence of extraction conditions on phenolic yields from pine bark: assessment of procyanidins polymerization degree by thiolysis", Food Chemistry., vol. 94, pp. 406-414, 2006.

[36] F. Martínez, "Obtención de taninos a partir de corteza de dos especies de pinos cubanos", Revista Forestal Baracoa., 3, (1), pp. 51, 1983.

[37] E. Aspé and K. Fernández, "The effect of different extraction techniques on extraction yield, total phenolic, and anti-radical capacity of extracts from Pinus radiata Bark", Industrial Crops and Products., vol. 34(1), pp. 838-844, 2011.

[38] W.S.G.R. Dvorak et al., "Pinus tecunumanii. In: Conservation and Testing of Tropical and Subtropical Forest Species by the CAMCORE Cooperative". College of Natural Resources, NCSU. Raleigh, NC. USA. pp: 188-209, 2000. 
[39] M. Mülek and P. Högger, "Highly sensitive analysis of polyphenols and their metabolites in human blood cells using dispersive SPE extraction and LC-MS/MS”, Anal Bioanal Chem., vol. 407, pp. 1885-1899, 2015.

[40] S. Hashim et al., "Rapid solid-phase extraction and analysis of resveratrol and other polyphenols in red wine", Journal of Chromatography A., vol. 1313, 25, pp. 284-290, 2013.

[41] J. Hellström, and P. Mattila, "HPLC Determination of Extractable and Unextractable Proanthocyanidins in Plant Materials", J. Agric. Food Chem., vol. 561, pp. 77617-7624, 2008.

[42] A. Romani et al.," Analysis of condensed and hydrolysable tannins from commercial plant extracts", Journal of Pharmaceutical and Biomedical Analysis., vol. 41, 2, pp. 415-420, 2006.

[43] G.E. Rohr, "Analytical investigation and isolation of procyanidins from Crataegus leaves and flowers". Tesis Doctoral. Swiss Federal Institute of Technology. Zurich, 1999.

[44] O. Yesil-Celiktas et al., "Determination of polyphenolic constituents and biological activities of bark extracts from different Pinus species", J. Sci. Food Agric., vol. 89, pp. 1339-1345, 2009.

[45] M. Jerez et al., "Procyanidins from pine bark: Relationships between structure, composition and antiradical activity"., Food Chemistry, vol. 104, (2), pp. 518-527, 2007b.

[46] K.J. Cho et al., "Inhibition mechanisms of bioflavonoids extracted from the bark of Pinus maritime on the expression of proinflammatory cytokines". Ann N Y Acad Sci. vol. 928, pp. 141-156, 2001.

[47] R.A. Sarria-Villa et al., "Extraction of peholic compounds from Pinus patula bark using etanol-water mixtures and the anti-inflammatory action of the ethanolic extract"., International Journal of Current Research, vol. 10 (3), pp. 66886-66895

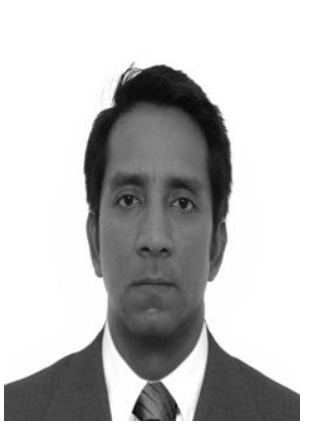

Rodrigo Andrés Sarria Villa. Investigador del Grupo de Investigación en Química Analítica Ambiental de la Universidad del Cauca y Profesor Ocasional tiempo completo del Departamento de Química de la Universidad del Cauca, Popayán, Colombia. Químico de la Universidad del Cauca, Popayán, Colombia. Maestría en Ciencias Químicas de la Universidad del Valle, Cali, Colombia y Doctor en Ciencias Químicas de la Universidad del Valle, Cali, Colombia, cuenta con 3 años de experiencia como docente, 10 años de experiencia como investigador y es miembro de la sociedad ambiental SETAC. Sus áreas de interés son: Química ambiental, toxicología y recursos renovables. ORCID: https://orcid.org/0000-0003-1295-7865.

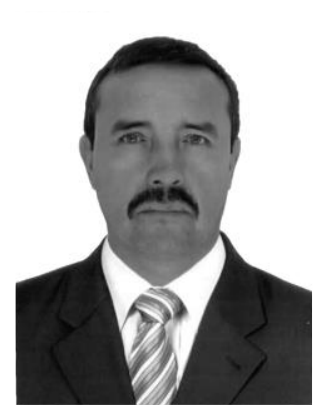

José Antonio Gallo Corredor. Investigador y Coordinador del Grupo de Investigación en Química Analítica Ambiental de la Universidad del Cauca, Popayán, Colombia. Licenciado en Ciencias de la Educación Química y Biología de la Universidad Pedagógica y Tecnológica de Colombia, Tunja, Colombia. Maestría en Ciencias Químicas de la Universidad del Valle, Cali, Colombia y Candidato a doctor en Ciencias de la Universidad del Cauca, Popayán, Colombia y cuenta con 20 años de experiencia como docente e investigador. Sus áreas de interés son: Contaminación por actividad minera y aplicaciones en residuos agroforestales. ORCID: https://orcid.org/0000-0002-4861-2837.

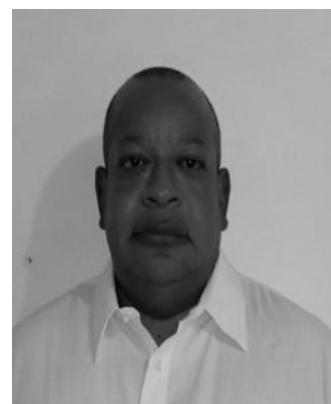

Ricardo Benítez Benítez. Investigador del Grupo de Investigación en Química de Productos Naturales de la Universidad del Cauca, Profesor Titular del departamento de Química de la Universidad del Cauca, Popayán, Colombia. Licenciado en Bioquímica por la Universidad del Valle, Cali, Colombia. Maestría en Ciencias Químicas de la Universidad del Valle, Cali, Colombia y Doctor en Ciencia y Tecnología de Alimentos de la Universidad de Lleida, Lleida, España y cuenta con más de 30 años de experiencia industrial, docente y de investigación. Sus áreas de interés son: Bioquímica, actividad enzimática y recursos renovables.

ORCID: https://orcid.org/0000-0002-8999-3680. 